\title{
A Conceptual Framework for Developing Indoor Spatial Choice Model
}

\author{
S. H. Cha and T. W. Kim
}

\begin{abstract}
Detailed space use analysis for a building is needed in the near future for building energy efficiency and effective space planning. In this regard, the development of an indoor spatial choice model is indispensable for better space use prediction. However, research efforts into the model are still insufficient in this area. As an initial study, this paper reviews spatial choice behavior, and proposes a conceptual framework that displays the necessary development steps, behavioral rules, and required data for developing indoor spatial choice model. Finally, future opportunities and challenges are discussed.
\end{abstract}

Index Terms - Occupant behavior, space use analysis, spatial choice model, space planning.

\section{INTRODUCTION}

A great amount of energy is consumed in building environments, and the human carbon footprint has rapidly increased around the world. A major concern over the last few decades has been how to curtail energy consumption in the building sector. Considerable research has been committed to devising energy-saving technologies in various fields, such as developing energy-efficient mechanical, electrical, and plumbing (MEP) systems, building envelopes, and eco-design Through these contributions, the goal of making buildings energy efficient attained a measure of success.

Research is still needed to achieve energy efficient buildings. Space use analysis is one of research areas that require more research efforts. A more realistic space use model in a building is needed as input for building simulation, building control, and space planning in the design phase. Most of research efforts for space use analysis in a building have focused on residential and office buildings in light of their own characteristics. For example, occupants in office buildings have one or a few designated workstations they usually stay in working days. These researches, accordingly, do not fit or require much extra work to be adjusted for other types of building in which spatial choices are existent, e.g., library. In addition, it is also true that digitalized and individualized work culture has been changing individual workspace use pattern from designated workstation to hot-desking: users select a workstation to stay for their own task. Thus, predicting spatial choice behavior as realistic as possible in a building become increasingly important.

Manuscript received November 10, 2013; revised February 13, 2014.

Seung Hyun Cha is with the Department of Architecture, the University of Cambridge, Cambridge, United Kingdom (e-mail: sc734@cam.ac.uk).

Tae Wan Kim are with the Civil and Environmental Engineering Department, Stanford University, Stanford, CA 94305 USA. He is now with the Department of Civil and Architectural Engineering, City University of Hong Kong, Hong Kong, The People's Republic of China (e-mail: taewkim@cityu.edu.hk).

\section{CuRRent OCCUPANCY AND Space USE Models}

For a decade, stochastic models to predict occupant behaviors have mainly been developed. Of them, Page's model [1] and Multiple Modules (MUMO) model [2] are the advanced versions for occupancy prediction by combining stochastic process with agents based modeling. Nevertheless, Page's model and MUMO model are more likely to be applicable to building control systems of existing buildings rather than space planning. The primary part of their models is transition probabilities for which empirical data of occupancy must be collected; when such data are unavailable, camera or detailed interview and survey, which can only be obtained from existing building, should be conducted. Even if enough data are accumulated so that architects or simulator can pick one type of occupant in the database through filed studies, it is not easy to extract the reasons for activities [3]. Accordingly, the generated occupancy pattern in a certain building has a lower applicability to other and other types of buildings with different purpose, size, design, etc. Kim [4] developed a framework for an automated activity-space mapping by systemizing the relationship between user, user activity and space. The primary concept of the framework is automatically to find a space type which meets the activity requirements, i.e. required equipment, size of room, etc., and the computation of the utilization of spaces is subsequently followed. This process assists client and architects' decision making for better design through iterative refinements of potential overloaded spaces. Albeit well defined framework with the formalized relationship between user, activity, and space, it is also not sufficient for where users are required to make spatial choices because it does not directly link activities with a space instance, i.e. a building is comprised of a number of space instances. On the contrary, activities are linked to a space type, which is comprised of a couple of space instances with similar properties. For this reason, this research has a limitation in displaying dynamic spatial choice realistically.

In User Simulation of Space Utilization (USSU) developed by Tabak [5], a nearest location from an initiator is chosen for a certain activity out of locations at which an activity type of abstract space - expressed by node and lines - matches with an activity type of activity. Goldstein [6] put an extension to USSU that a location far from the initiator may be selected with a lower probability according to a cost function. It seems to be unrealistic assuming that individual spatial choice behavior, subject to several parameters, is explained only by distance. In office building where users have one or a few designated workstations, this assumption might be enough to realize the space-use analysis, but it is self-evident that other parameters that may affect individual spatial choice pattern 
must be taken into account where individuals' single spatial choices mostly compose space use in a building. To our knowledge, there is no solid model which considers such parameters systematically for indoor spatial choice behavior.

A more comprehensive, general, and detailed space use model is needed in the near future to generate a more realistic input data for building simulation and support better design solution in terms of space planning. As the indoor space is ultimately designed for user to conduct their various activities, user, user activity and space are the three essential components of space use model. We believe that the next generation model will be developed based on formalized relationships between user, user activity, and space, and the well-organized properties of the respective components so that it can be applicable to other buildings with diverse contexts in the design phase [4]. In this regard, the development of an indoor spatial choice model is an integral part of space use model for better prediction. Spatial choice behavior is briefly reviewed in the next section. Section IV proposes a conceptual framework for indoor spatial choice model.

\section{Spatial ChOICE BehaVior}

Individuals implement their spatial choices based on the outcomes they perceive, interpret, evaluate, and compare in the environments through their cognitive and affective standards. Individuals try to maximize their benefits or satisfaction level in situations necessary for choosing one of available alternatives. In the early years, a decision maker was regarded as a person pursuing an optimal decision with perfect rationality and with full information on alternatives for the environments based on rational choice theory.

In reality, however, the concept was not well connected with actual spatial choice behaviors of building users. It is because individuals' spatial choices are much more complicated and extensive, needing to take into account several factors such as social, psychological and physical human behavioral aspects, individual heterogeneity, and spatial complexities and interactions. Those have been handled in many disciplines concerning a more sophisticated and accurate spatial choice model. In the psychology discipline, attention has been concentrated on spatial cognition matters (i.e., how people process spatial information) and then on distinct spatial layouts formulated for each individual, called mental map [7] On the other hand, geographers have been interested in the effects of spatial interaction on spatial choice [8].

Extensive work has thus been devoted to spatial choice behavior models in the areas of shopping location, leisure and recreation choice, facility location, residential location choice, and migration modeling Spatial choice models have, however, focused mainly on urban-scale choices, which must be considered separately from spatial choice on building scale. For example, Boots [9] regard distance as the main index determining inter metropolitan migration decisions, whereas it is not certain how important distance is on the building level Even though distance is considered a main parameter in evacuation simulation models in a building [10], evacuation models are for special circumstances, which are different from general choice circumstances. For that reason, indoor spatial choice must be discussed separately.

We postulate that disaggregate single spatial choices demonstrate the use of entire building. In only one day, people encounter many situations when they choose a seat or table at a small level, and a room, a space instance in this paper at large level, in a building for any given reason such as which restroom and cafeteria they use, then a seat in the cafeteria, or studying space in a library building. We assume that chosen spaces are basically on the ground of each individual's utility maximization pattern based on personal preferences and intentions.

Furthermore, spatial choice cannot be explained by such process only, because there are the substantial aforementioned factors that can be described in detail, such as habitual behavior, satisficing rule (good enough), bounded rationality, variety seeking, familiarity, inflow of new information, environmental and social constraints, spatial correlation, difference, and competition, all largely influencing spatial choice pattern and needing to be accounted for.

\section{A CONCEPTUAL FRAMEWORK FOR INDOOR SPATIAL CHOICE MODEL}

A conceptual framework of indoor spatial choice model is proposed in this section. An individual choose a space out of a set of relevant space instances for a certain activity to maximize one's utility. Fig. 1 shows the necessary steps, behavioral rules, and required data for developing indoor spatial choice model in consideration of its own characteristics. This section comprehensively elucidates each component of Fig. 1.

\section{A. Random Utility Theory}

Choice models are generated based on a statistical model whose underlying theory is random utility theory. The main idea of random utility theory is that an individual choose an alternative with the highest utility out of several alternatives that meet their purposes. In this theory, each alternative (a space instance in a building) will have its own attributes and measure of the attributes for a certain activity. Generally, researchers may not be able to understand all the possible attributes affecting choice outcome. Therefore, total utility $U_{i j}$ in case individual $i$ choose space $j$ is comprised of a deterministic utility $V_{i j}$, and a unobserved utility $\varepsilon_{\mathrm{ij}}$ as seen in equation (1) [11]. The deterministic component $V_{i j}$ is calculated

$$
U_{i j}=V_{i j}+\varepsilon_{i j}
$$

understanding attributes(e.g. crowdedness, noise level) and their measures of each space instance. The measures are weighted to mirror different importance of each attribute according to a certain activity and individual. The unobserved utility $\varepsilon_{i j}$ is described by a probabilistic distribution. For example, a probit model assumes that unobserved utility $\varepsilon_{i j}$ is normally distributed. A statistical model that is well suited to indoor spatial choice behavior needs to be identified since different statistical models have different assumptions for the 
probabilistic distribution.

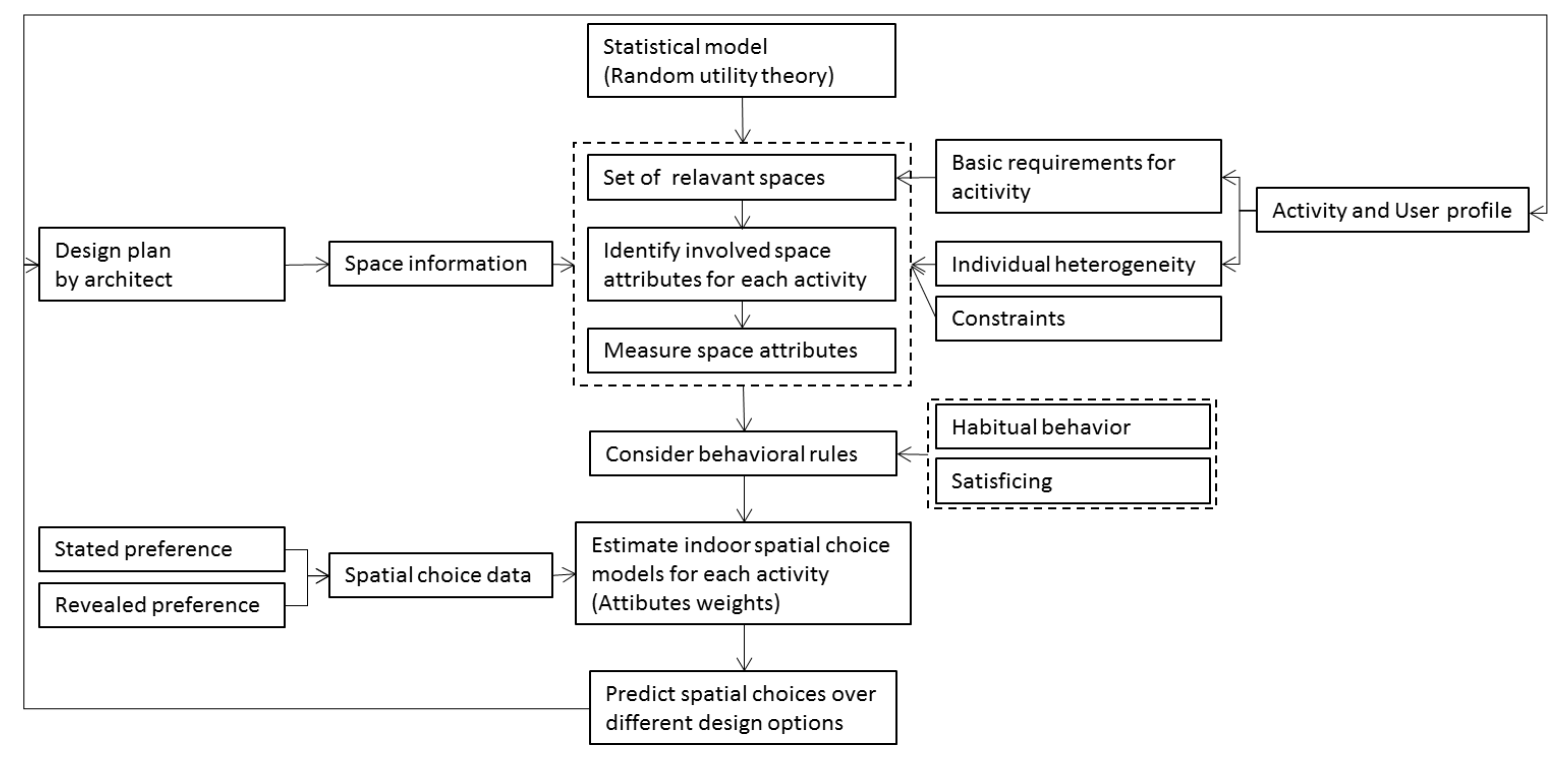

Fig. 1. A conceptual framework for indoor spatial choice model.

\section{B. Individual Heterogeneity}

Not all individuals who face a certain choice in situation behave alike, a fact that results from individual heterogeneity. People have dissimilar cultural, educational, regional, and social backgrounds, leading to heterogeneous socio-demographic and intrinsic characteristics. An individual who knows more about or is more familiar with a certain building may choose to use a space that is more suited to his or her lifestyle. Learning and information-processing ability also influence choice behavior when an individual visits an unfamiliar building. It is essential, therefore, to answer how individual heterogeneity should be represented in indoor spatial choice model.

Categorizing occupants in detail by their varying characteristics with particular attributes is the way of achieving a more reliable occupant behavior model. On a building scale, for instance, Liao [12] groups users into primary users and secondary occupants according to occupation in order to discern the length of stay of occupants. Tabak [5] also uses personal data, like roles, organizational unit, and FTE (full-time equivalence), to model an activity-based occupancy model. Fujii [13] displays an interest in interaction between the architectural environment and human action based on a human's belief and knowledge. He suggests that human action cannot be explained by only the environment itself, but by the environment perceived and cognized by an occupant, intention, belief and knowledge. On the basis of this concept, he creates a simulation model showing different thermal occupant behaviors according to personal internal attributes. Even though the examples are not directly related to indoor spatial choice behavior, it is worthy of attention that individual heterogeneity causes users' different behavior patterns in a building.

\section{Data Requirements}

Four main data sets are needed for developing indoor spatial choice model: a set of attributes involved to indoor spatial choice for each activity, space information, activity and user profile, and spatial choice data. Space information and activity and user profile are provided by architects according to their design plan and estimation of what activities and users will be in the building.

First, space information represents measures of each attribute in space instances according to building design. For example, a measure of distance from entrance of a certain space instance in a building is obtained from space information.

Second, activity profile means the details of all activities estimated for a certain building: activity type, and basic requirements for activity, e.g., necessary equipment, minimum space size. Therefore, activity profile comes up with a relevant space set for each activity, which is alternatives for indoor spatial choice, narrowed down from an entire space set in a building.

Third, two types of statistical methods are available to obtain spatial choice data in terms of the means of collecting data: one is stated preference method based on hypothetical choice situations generated by experiment modeler, and the other is revealed preference method based on actual choice situation. Both methods have their own strengths. The most significant difference between two methods are variability and validity. In case of stated preference methods, researchers can easily estimate attributes by means of hypothetically giving different scenarios with diverse alternatives but there may be some doubt about the choices themselves since choice situations are virtually fabricated. It is a possible case that a respondent answers to choose alternative $\mathrm{A}$ in a hypothetical choice situation, but alternative $\mathrm{B}$ in reality. On the other hand, revealed preference method has no doubt about the choices themselves, but to the contrary, cannot consider anticipated scenarios since it is from actual data.

Lastly, a set of attributes relative to indoor spatial choice must be identified. To our knowledge, however, indoor spatial choice model in light of spatial information has not been explored unlike other declines, e.g. transportation [14], [15] and recreation[16], in which several attributes are already discovered so that other researchers can employ or refer to them. Therefore, a set of attributes for indoor spatial 
choice must be first researched and generated for developing indoor spatial choice model. Although some important attributes are partly discovered for seat selection in the library [17], [18], they are not general enough for indoor spatial choice model

In order to comprehend involved attributes with indoor spatial choice model, we hypothesize that main attributes are varying according to user type and activity type since each activity and user have their own characteristics. For example, an activity of reading article may prefer a quiet and relatively small space whereas a group meeting may pay less attention to noise level but require a big space. In this regard, noise level will be a main attribute for reading article, but not for a group meeting.

\section{Behavioral Rules}

Behavioral rules must be taken into account in indoor spatial choice model since it can lead to a huge difference in choice estimation. Among many of behavioral rules, only habitual behavior and satisficing are discussed since we believe these two are main factors for indoor spatial choice behavior.

\section{1) Habitual behavior}

Psychologically, decision makers judge situations through one of the following two distinct cognitive systems, known as the dual-process theory [19]: intuitive and reflective systems based on their speed, controllability, and the contents of cognitive process in Fig.2. System 1 is fast, and activated on simple heuristic rules, whereas System 2 is slower and goes through more substantial monitoring processes. System 1 is explained with habitual behavior, and System 2 concerns the optimization process, considering all possible alternatives. The influences of the two systems vary in personal characteristic, given tasks, the individual's mood and the individual's intelligence.

In a given choice situation, people show a propensity to make repetitive choices. This pattern can be explained by an optimal choice through full evaluation stages, otherwise by habitual behavior. Habitual behavior means to re-apply the same choice in identical or similar situations. This behavior avoids additional search efforts, time loss, and cognitive cost by reusing the same choices that individuals experienced, and were satisfied with in the past [20].

Habitual behavior ultimately means limited knowledge about the environment and poor awareness of better alternatives. Deliberate actions are distinguished from habitual behavior in that these need a certain motivation for choices, such as perilous results and substantial benefits, and have enough time for the decision-making process [19]. The frequency of habitual behavior differs by the type of behavior, individual, and environment. In a consumer choice study, Swait [21] described that the degree of recurrence over the set of goods is diverse - for example, milk, margarine, and butter show around $50 \%$ of routine choice, whereas toothbrushes, deodorants, and cereals shows less than $20 \%$ of routine choice.

Thus, it can be inferred that the frequency of habitual behavior of individual's spatial choice in a building also varies over building type, activity, building design, and individual's characteristics, thereby affecting overall occupancy pattern. Intuitively.

\begin{tabular}{|cc|}
\hline System 1(Intuitive) & System 2 (Reflective) \\
Process characteristic & Controlled \\
Automatic & Effortful \\
Effortless & Deductive \\
Associative & Slow, serial \\
Rapid, parallel & Self-aware \\
Process opaque & Rule application \\
Skilled action & Neutral \\
Content on which processes act \\
Affective & Statistics \\
Causal propensities & Abstract \\
Concrete, specific & Sets \\
Prototypes & \\
\hline
\end{tabular}

Fig. 2. Two cognitive systems [19].

\section{2) Satisficing}

Simon [22] suggested that humans implement decision processes under bounded rationality. Decision makers cannot evaluate all the alternatives, and therefore choose an alternative which meet their acceptability threshold based on their knowledge. This phenomenon occurs more often in complicated circumstances and information overload situations. Humans follow simple rules when facing complicated circumstances, thereby choosing an alternative they face at the early stage of a search process, often called a premature termination of search for evidence. In this regard, this choice behavior conflicts with an optimal choice based on random utility theory.

These patterns can be explained with evading the cognitive cost because decision makers cannot assure the degree of obtainable benefits by choosing other alternatives through the search process. Decision makers undergo the procedures to estimate the gap between cognitive costs and benefits at all stages of decision making. The trade-off between them is the important determinant for whether putting in more effort is useful. Indoor spatial choice behavior needs to be explained by satisficing theory. It is intuitively true that an occupant picks a space instance which meet minimum requirements for one's activity without exploring all possible space instances in an entire building. However, it is not clear yet that satisficing theory to what extent will be influential on indoor spatial choice behavior. The degree of satisficing impact can vary over size of building, activity type, and user type. For example, the building users' search effort will diminish as the size of building expands.

\section{3) Constraints}

Another factor influencing choice behavior is a variety of constraints that limits the individual's choice on attitude and preference. Also in the spatial behavior context, human subjectivity is restricted by constrains of the physical and social environments. Desbarats [23] developed four stages for narrowing a possible set of alternatives from top to bottom by external and internal constraints in Fig. 3.

In the first stage, the objective choice set is set by environment. Individual spatial behavior takes place on the choice set given by the environment. Institutions regulate the 
available alternatives in a choice set by controlling the supply of opportunities. The effective choice set is determined by the lack of information and social pressure. Unrecognized existing options contract a set of choice options, and less education by an individual may influence the lack of information. In addition, social pressure limits some possible options on a choice set due to the need of decision makers to conform to social norms. The impact on social pressure varies with personal traits, thereby resulting in different effective choice sets by individuals.

Preferences and attitudes of an individual are molded by societal values, so effective choice set is transformed to destination choice not corresponding to personal values. Finally, situations may block the choice behavior, that is, the institutional gatekeeper may interrupt the execution of socially undesirable behavior, or limitation of occupancy may have the decision maker not choose options. Many constraints must be considered for indoor spatial choice model for more accurate prediction. A certain space will be allowed to a certain users and time, e.g. staff-only space, meeting space with opening hours.

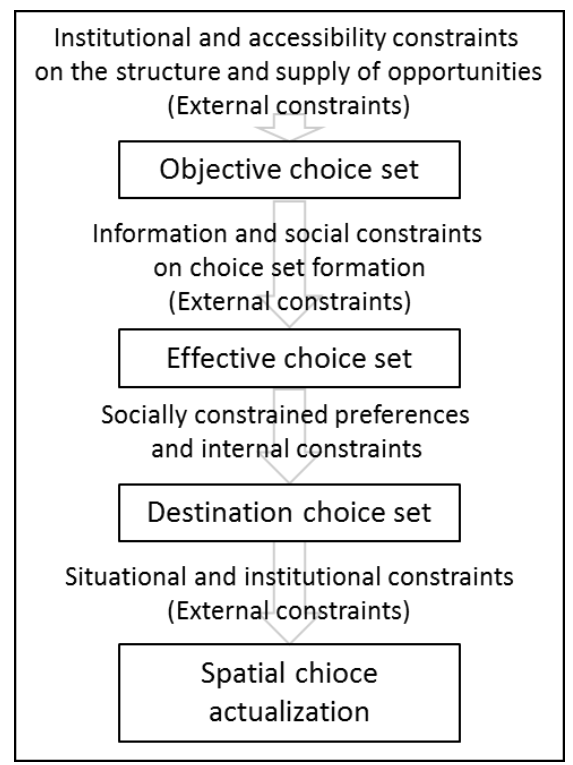

Fig. 3. Internal and external constraints on movement behavior [23].

\section{DISCUSSION}

Individuals implement their spatial choices to maximize their benefits or satisfaction level in situations necessary for choosing one of available alternatives. Indoor spatial choice behavior can be well explained by a choice model. The advantage of employment of a choice model is that information of probabilistic prediction of spatial choice is provided to building designers. Accordingly, this information can be utilized in design stage to forecast spatial choice distribution according to different design options.

However, there are still some challenges in developing an indoor spatial choice model. First, a set of attributes relative to indoor spatial choice must be identified, which are hypothesized that a set of attributes vary over activity and user type. Second, devising reasonable measures of each attribute are also a demanding part. For example, supposing space aesthetics is one of main attributes of reading activity for spatial choice, it will not be a simple job to set up an objective measure for it. Lastly, application of behavioral rules to a choice model requires many assumptions that might lead to wrong predictions. Research about indoor spatial choice behavior is at an early stage, and therefore there is not enough information to establish valid and underlying assumptions for behavioral rules for developing indoor spatial choice model.

For the stated challenges, research efforts are needed to develop indoor spatial choice model as the necessity and benefit of indoor spatial choice model is evident.

\section{ACKNOWLEDGMENT}

This research received no specific grant from any funding agency in the public, commercial, or not-for-profit sectors.

\section{REFERENCES}

[1] J. Page, D. Robinson, N. Morel, and J. Scartezzini, "A generalised stochastic model for the simulation of occupant presence," Energy Build., vol. 40, no. 2, pp. 83-98, 2008.

[2] C. Liao, Y. Lin, and P. Barooah, "Agent-based and graphical modelling of building occupancy," J. Build. Perform. Simul., vol. 5, no. 1 , pp. 5-25, 2012

[3] G. Zimmermann, "Modeling and simulation of individual user behavior for building performance predictions," in Proc. 2007 summer Comput. Simul. Conf. Calif., pp. 913-920, 2007.

[4] T. W. Kim, R. Rajagopal, M. Fischer, and C. Kam, "A knowledge-based framework for automated space-use analysis," Autom. Constr., pp. 1-12, Sep. 2012.

[5] V. Tabak, "User Simulation of Space Utilisation," Ph.D. thes Technische Universiteit Eindhoven, 2008.

[6] R. Goldstein, A. Tessier, A. Khan, and K. S. East, "Space layout in occupant behavior simulation comparison with related work," pp. 14-16, 2011.

[7] T. Gärling, A. Böök, and E. Lindberg, “Cognitive mapping of large scale environments: the interrelationship of action plan, acquisition and orientation," Environment and Behavior, vol. 16. pp. 3-34, 1984

[8] A. S. Fotheringham, T. Nakaya, K. Yano, S. Openshaw, and Y. Ishikawa, "Hierarchical destination choice and spatial interaction modelling: a simulation experiment," Environment and Planning,vol. 33, pp . 901-20, 2001.

[9] B. N. Boots and P. S. Kanaroglou,'Incorporating the effects of spatial structure in discrete choice models of migration," Journal of Regional Science, vol. 28, pp. 495-509, 1988.

[10] S. M. Lo, Z. Fang, P. Lin, and G. S. Zhi, "An evacuation model: the SGEM package,” Fire Saf. J., vol. 39, no. 3, pp. 169-190, Apr. 2004.

[11] M. Ben-Akiva and S. Lerman, Discrete Choice Analysis: Theory and Application to Travel Demand, MIT Press, 1985.

[12] C. Liao, Y. Lin, and P. Barooah, "Agent-based and graphical modeling of building occupancy," J. Build. Perform. Simul., vol. 5, no. 1, pp. $1-25,2010$.

[13] H. Fujii and J. Tanimoto, "Integration of building simulation and agent simulation for exploration to environmentally symbiotic architecture," Build. Environ., vol. 39, no. 8, pp. 885-893, Aug. 2004.

[14] D. Ettema, T. Arentze, and H. Timmermans, "Social influences on household location, mobility and activity choice in integrated micro-simulation models," Transp. Res. Part A Policy Pract., vol. 45, no. 4, pp. 283-295, May 2011.

[15] E. Cherchi, "A mixed logit mode choice model on panel data: accounting for systematic and random variations on responses and preferences," Transp. Res., pp. 1-20, 2008.

[16] L. M. Hunt, "Recreational Fishing Site Choice Models: Insights and Future Opportunities," Hum. Dimens. Wildl., vol. 10, no. 3, pp. 2005.

[17] K. M. Webb, M. A. Schaller, and S. A. Hunley, "Measuring Library Space Use and Preferences: Charting a Path Toward Increased Engagement," portal Libr. Acad., vol. 8, no. 4, pp. 407-422, 2008.

[18] R. Applegate, "The Library Is for Studying," J. Acad. Librariansh., vol. 35, no. 4, pp. 341-346, Jul. 2009.

[19] D. Kahneman and S. Frederick, "Heuristics and biases: The psychology of intuitive judgment," Representativeness Revisited: Attribute Substitution in Intuitive Judgment, in T. Gilovich, D. Griffin, \& D. Kahneman (Eds.), Cambridge University Press, 2002. 
[20] P. Goodwin, R. Kitamura, and H. Meurs, "Some principles of dynamic analysis of travel behaviour," Developments in Dynamic and Activity-Based Approaches to Travel Analysis, pp. 56-72, 1990.

[21] W. L. Adamowicz and J. D. Swait, "Are food choices really habitual? Integrating habits , variety seeking and compensatory choice in a utility maximizing framework," American J. of Agricultural Economics, vol. 95, no. 1, pp. 17-41, 2013.

[22] H. A. Simon, "Behavioral model of rational choice," Quart. J. Econom. vol. 69, pp. 99-118, 1955.

[23] J. Desbarats, "Spatial Choice and Constraints on Behavior," Annals of the Association of American Geographers, vol. 73, no. 3, pp. 340-357, 1983.

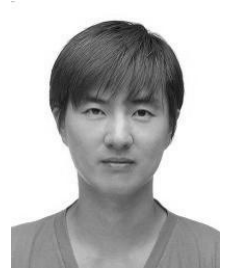

Seung Hyun Cha was born and grew up in Seoul, South Korea. He received B.E. degree in architectural engineering from Korea University and M.S. degree in sustainable design and construction from Stanford University, USA. He is currently a doctoral student in the Department of Architecture at the University of Cambridge, UK.

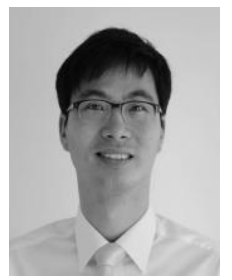

Tae Wan Kim is from Seoul, South Korea. He earned his Ph.D. in 2013 in civil and environmental engineering from Stanford University, California, USA. He received his B.S. in engineering from Seoul National University. He has four years of construction management experience in Korea. He is currently an Assistant Professor in the Department of Civil and Architectural Engineering at City University of Hong Kong in Hong Kong. His research employs the framework of Virtual Design and Construction (VDC) to explore and formalize the relationships between building users and designed space, commonly represented in a Building Information Model (BIM), with the goal of enhancing performance analyses to better understand the operation of a building. 